Short Communication

Genomics and Bioinformatics

\title{
SSD - a free software for designing multimeric mono-, bi- and trivalent shRNAs
}

Gabriel José de Carli $^{1,4}$ iD, Abdon Troche Rotela ${ }^{2,3}$ (D), Greice Lubini ${ }^{4}$ iD, Danyel Fernandes Contiliani ${ }^{1,4}$ (D), Nidia Benítez Candia ${ }^{2}$ (D), Thiago S. Depintor ${ }^{1}$ (D), Fabiano Carlos Pinto de Abreu ${ }^{1,4}$ (D), Zilá Luz Paulino Simões $^{1,4}$ (D) , Danilo Fernández Ríos ${ }^{2}$ (iD) and Tiago Campos Pereira ${ }^{1,4}$ iD

${ }^{1}$ Universidade de São Paulo, Faculdade de Medicina de Ribeirão Preto, Departamento de Genética, Ribeirão Preto, SP, Brazil.

${ }^{2}$ Universidad Nacional de Asunción, Facultad de Ciencias Exactas y Naturales, San Lorenzo, Paraguay.

${ }^{3}$ Universidad Nacional de Asunción, Facultad Politécnica, San Lorenzo, Paraguay.

${ }^{4}$ Universidade de São Paulo, Faculdade de Filosofia, Ciências e Letras de Ribeirão Preto, Departamento de Biologia, Ribeirão Preto, SP, Brazil.

\begin{abstract}
RNA interference (RNAi) is a powerful gene silencing technology, widely used in analyses of reverse genetics, development of therapeutic strategies and generation of biotechnological products. Here we present a free software tool for the rational design of RNAi effectors, named siRNA and shRNA designer (SSD). SSD incorporates our previously developed software Strand Analysis to construct template DNAs amenable for the large scale production of mono-, bi- and trivalent multimeric shRNAs, via in vitro rolling circle transcription. We tested SSD by creating a trivalent multimeric shRNA against the vitellogenin gene of Apis mellifera. RT-qPCR analysis revealed that our molecule promoted a decrease in more than $50 \%$ of the target mRNA, in a dose-dependent manner, when compared to the control group. Thus, SSD software allows the easy design of multimeric shRNAs, for single or multiple simultaneous knockdowns, which is especially interesting for studies involving large amounts of double-stranded molecules.
\end{abstract}

Keywords: Gene silencing, siRNA, multimeric, shRNA, free software.

Received: September 19, 2019; Accepted: January 13, 2020.

RNA interference (RNAi) is a gene silencing technology (Fire et al., 1998) with broad applications, from reverse genetics and functional genomics (Suzuki et al., 2018), to treating diseases (Matthiesen et al., 2019), combating cancer (Ganesh et al., 2018), generating animal models (Guerreo-Rubio et al., 2019) and biotechnological products (Metwali et al., 2015).

The effector molecule is a short RNA duplex, composed of two strands of approximately 21 nucleotides, with two overhanging bases at the 3' end (Elbashir et al., 2001). However, several types of precursors can trigger gene silencing, such as long double-stranded RNAs (dsRNAs) from 200 to 800 base pairs (bp) (Guo et al., 2018), hairpin RNAs (hpRNAs) (Hussain et al., 2019), small interfering RNAs (siRNAs) (Guo et al., 2018), short hairpin RNAs

Send correspondence to Tiago Campos Pereira. Universidade de São Paulo, Faculdade de Medicina de Ribeirão Preto, Departamento de Genética, Ribeirão Preto, SP, Brazil. Laboratório de Genética Molecular da Anidrobiose, Sala 13, Bloco 14 - Departamento de Biologia, Faculdade de Filosofia, Ciências e Letras de Ribeirão Preto FFCLRP, Universidade de São Paulo - USP, Av. Bandeirantes, 3900.Bairro Monte Alegre. Ribeirão Preto, SP, Brazil.
(shRNAs) (Wang et al., 2018), among others. Notoriously, an ingenious and inexpensive way to produce shRNAs in large quantities was developed, via in vitro transcription of a circularized DNA template, generating multimeric shRNAs (mshRNAs) (Seyhan et al., 2006; Abe et al., 2012). In the last decade, such multimeric shRNAs generated by rolling circle transcription have been used by other groups and have been shown to be an interesting option for RNAi (Wang et al., 2015; Shopsowitz et al., 2016; Wu et al., 2016; Kim et al., 2018).

Here, we present a software tool for a fast and rational design of siRNAs and mshRNAs named "siRNA and multimeric shRNA designer" (SSD) (Figure 1). SSD incorporates the siRNA design tool from our previously developed software Strand Analysis (Pereira et al., 2007), since the design of mshRNAs requires siRNA sequences as input. Once the siRNA duplex is determined, SSD can generate a DNA sequence (the template) whose transcription will result in the mshRNA. There are three options of mshRNAs (Figure 2). The monovalent mshRNA (Figure 2A) displays only one silencing sequence and is a molecule based on the original mshRNA, as designed by Seyhan et al. (2006). The 


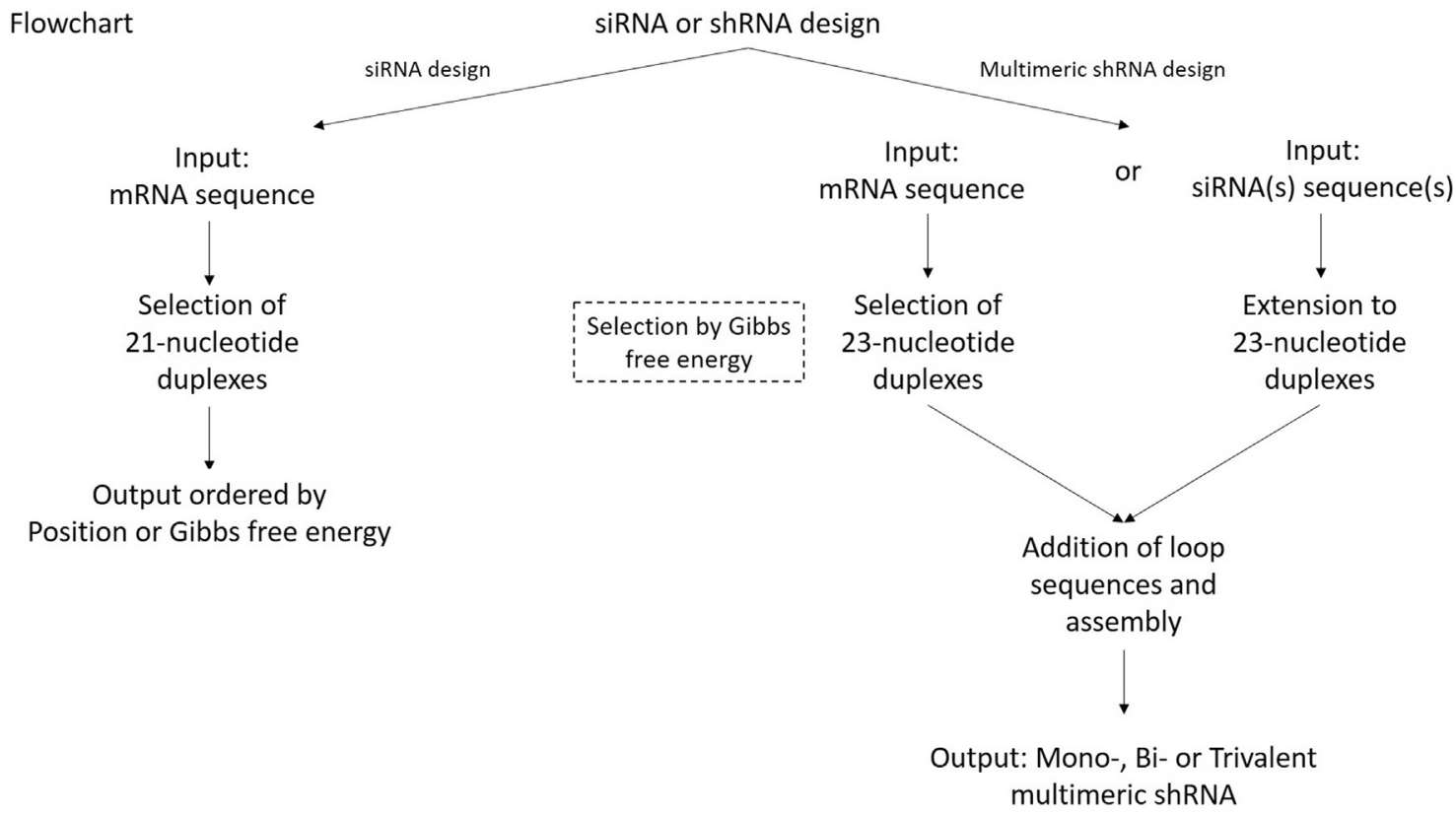

Figure 1 - si- and shRNA (SSD) flowchart. SSD software is suitable for designing both siRNAs and multimeric shRNAs. For siRNA design, a target mRNA sequence must be used as 'input', while for mshRNA design, either the target mRNA or the previously designed siRNAs sequences can be used as 'input'. siRNAs may be selected either by 'position' within the target mRNA or by 'Gibbs free energy' (i.e., silencing efficiency).

bivalent mshRNA contains two silencing sequences (Figure 2B); and the trivalent, three (Figure 2C). Both bi- and trivalent mshRNAs are suitable for silencing single genes by targeting different sites within the mRNA, thus increasing the chance of knockdown. Alternatively, transcripts from different genes can be targeted with the same mshRNA, enabling double or triple knockdown by bi-/trivalent mshRNAs, respectively.

In order to make the shRNA design as simple as possible, SSD offers an integrated option to generate the mshRNA template by using the messenger RNA (mRNA) as input. In such case, SSD will select the three most func-

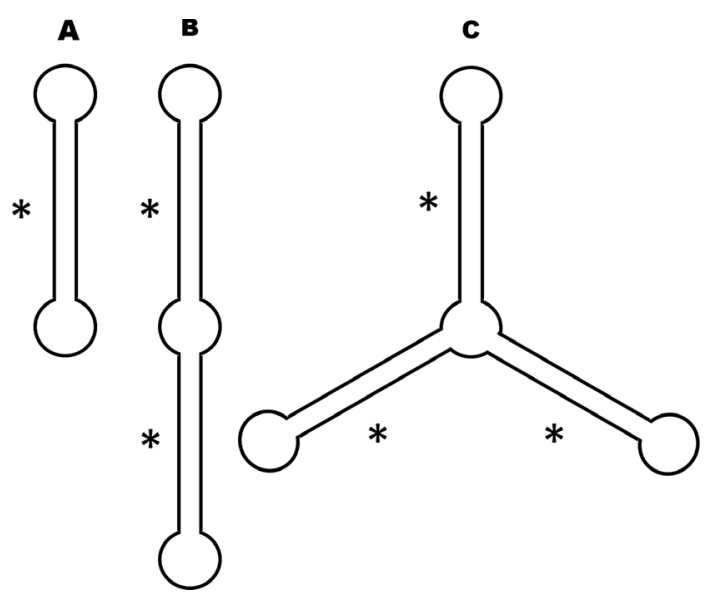

Figure 2 - Predicted secondary structures of DNA templates for multimeric shRNAs, designed by SSD against the Apis mellifera vitellogenin gene. Circularized templates for mono- (a), bi- (b) and trivalent mshRNAs (c). Each base paired region $(*)$ corresponds to a different shRNA. tional and non-overlapping siRNAs to design all the three possible mshRNAs: mono-, bi- or trivalent.

The programming language used to build SSD was Python version $3.5 \mathrm{x}$, because it is a high-level, general-purpose and easily interpreted language. Furthermore, Python has a large, user-friendly standard library, automatic memory management, and dynamic features. The graphical interface tool used was PyQt5, since it provides a high number of prebuilt functions. Moreover, PyQt5 is widely used and has a considerable amount of documentation. One last advantage of Python is that SSD can be executed in most used operating systems (Windows, Mac, and Linux). The URL to download SSD, its requirements and a tutorial detailing how to use the software are on the web pages: https://github.com/bioinf2019/RNA_Tools and https://youtu.be/7pfQ7EVX5w8.

In order to validate SSD functionality, we designed trimeric mshRNAs against the Apis mellifera vitellogenin gene (NCBI accession number: NM_001011578.1) and Green Fluorescent Protein (GFP) (NCBI accession number: X83959.1); the latter was used as negative control. The designed linear DNA templates (183-mer) were custom synthesized (Exxtend - Soluções em Oligos, Brazil) and circularized via ligase reactions (20 ng of DNA, $1 \mathrm{U}$ of T4 DNA ligase (Promega), in a $10 \mu \mathrm{L}$ final volume reaction, overnight at room temperature). Subsequently, the entire ligation reaction volume was used for in vitro transcription, via TranscriptAid T7 High Yield Transcription Kit (Thermo Scientific), in a final reaction volume of $50 \mu \mathrm{L}$, for 4 hours at $37^{\circ} \mathrm{C}$. In order to remove the excess of free nucleotides after transcription, two precipitation steps with 
ammonium acetate $(5 \mathrm{M})$ were performed. One microliter of mshRNA solution was injected in young adult bees $(\mathrm{n}=$ 6-8) between the third and fourth tergites in the abdomen with a microsyringe (Hamilton), in three different concentrations, (i) $250 \mathrm{ng} / \mu \mathrm{L}$, (ii) $500 \mathrm{ng} / \mu \mathrm{L}$ and (iii) $2500 \mathrm{ng} / \mu \mathrm{L}$. After injection, bees were maintained at $34^{\circ} \mathrm{C}, 80 \%$ moisture and unrestricted food (mix of sugar, honey and pollen).

Five days later, total RNA was extracted with Trizol ${ }^{\circledR}$ and $1 \mu \mathrm{g}$ of RNA was used for reverse transcription reactions (SuperScript II ${ }^{\circledR}$, Thermo Fisher Scientific), using oligo $(\mathrm{dT})_{12-18}$ primers (Thermo Fisher Scientific). The cDNA was used in qPCR reactions (qPCRBIO SyGrenn Mix - PCRBIOSYSTEMS) to evaluate the relative expression of the vitellogenin mRNA. The qPCR was performed with the following conditions: $2 \mathrm{~min}$ at $95{ }^{\circ} \mathrm{C}$ followed by 40 cycles of $5 \mathrm{~s}$ at $95^{\circ} \mathrm{C}$ and $25 \mathrm{~s}$ at $60^{\circ} \mathrm{C}$. The dissociation cycle for every primer pair was from $95^{\circ} \mathrm{C}$ to $60^{\circ} \mathrm{C}(15 \mathrm{~s}$ each degree $), 60{ }^{\circ} \mathrm{C}(1 \mathrm{~min})$, and from $60{ }^{\circ} \mathrm{C}$ to $95^{\circ} \mathrm{C}(15 \mathrm{~s}$ each degree). Vitellogenin specific primers were: GCAGAATACATGGACGGTGT (forward) and GAACAGTCTTCGGAAGCTTG (reverse). As internal control, the gene RpL32 (Lourenço et al., 2008) was used with specific primers: CGTCATATGTTGCC AACTGGT (forward) and TTGAGCACGTTCAACAAT GG (reverse). $\mathrm{qPCR}$ results demonstrate that the trivalent mshRNA designed by SSD was effective in reducing the amount of vitellogenin mRNA in more than 50\% (compared to GFP group) when 500 ng of mshRNA were administered (Figure 3). Importantly, $250 \mathrm{ng}$ of mshRNA did not promote efficient knock down, while 2500 ng promoted unspecific effects (data not shown). Such results demonstrate the effectiveness of the current version of SSD, which will be updated once a year, to ensure continuous functionality and improvements.

Previously, Gvozdeva and colleagues developed a trimeric small-interfering RNA (tsiRNA), which is a linear duplex of RNA with $63 \mathrm{bp}$, composed of three sequential siRNAs (Gvozdeva et al., 2017). However, due to the im-

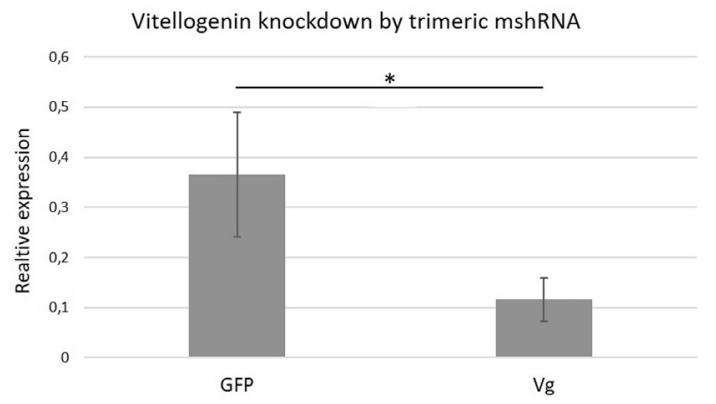

Figure 3 - Gene silencing mediated by trivalent multimeric shRNA designed by SSD. GFP group was injected with $500 \mathrm{ng}$ of mshRNAs against GFP gene (negative control); $\mathrm{Vg}$ group was injected with $500 \mathrm{ng}$ of mshRNAs against Apis mellifera vitellogenin. All experiments were performed in 6-8 biological replicates, each one composed of technical triplicates. Statistical difference was evaluated by ANOVA, with Student T test as post-hoc (* means $p<0.05)$. pressive yields of in vitro transcription systems (up to $7 \mathrm{mg}$ of transcripts per kit), mshRNAs are much cheaper than tsiRNAs, especially in studies using high amounts of RNA duplexes.

Moreover, due to its size ( $>30 \mathrm{bp}$ ) tsiRNA triggers interferon responses in mammalian cells. Thus, synthesis of tsiRNAs requires modified bases to avoid such unintended secondary effects, which increases the cost of synthesis. In order to be used in mammalian systems, our mshRNAs also demands an additional step - a phosphatase treatment to remove 5' triphosphate inserted during in vitro transcription (Kim et al., 2004). However, such treatment is much cheaper than using modified bases.

Another recent trimeric silencing RNA is the 'Y-RNA' (Jang et al., 2018). The structure of Y-RNA is very similar to our trimeric mshRNA (Figure 2C), due to a shared method of synthesis. A circular DNA template undergoes rolling circle transcription by T7 RNA polymerases, generating a long single-stranded RNA with the same secondary structure of our trimeric mshRNA (Figure 2C). However, an additional step is needed - a treatment with RNase $\mathrm{H}$ in the presence of an ssDNA helper, in order to promote the cleavage of RNA loops on the edge of the Y-RNA in a site-specific way. According to the authors, the RNase H step facilitates the cleavage of Y-RNA by the nuclease Dicer (Jang et al., 2018). Despite the similarities, the authors do not provide an easy method for a fast and rational design of Y-RNAs; moreover, the RNase $\mathrm{H}$ treatment makes the process much more laborious and more expensive when compared to the SSD approach.

In summary, we developed a free software tool (SSD) for rapid design of siRNAs, mono-, bi- and trivalent multimeric shRNAs, which can be easily synthesized in the laboratory, for single or multiple gene knockdowns. An online version of the software will be released shortly.

\section{Acknowledgments}

This work was supported by CAPES (Coordenação de Aperfeiçoamento de Pessoal de nível Superior - Finance Code 001), CNPq (Conselho Nacional de Desenvolvimento Científico e Tecnológico - Project: 832364/1999-1, Process: 131446/2018-5), FAPESP (Fundo de Amparo à Pesquisa do Estado de São Paulo - Process 2016/06657-0) and FACEN-UNA (Facultad de Ciencias Exactas y Naturales, Universidad Nacional de Asunción - PIC-48-2018).

\section{Conflict of Interest}

The authors declare that there is no conflict of interest associated with this study.

\section{Author Contributions}

All authors contributed with this research. GJC, TCP and GB conceived the software; ATR, NBC and DFR developed the software script; DFC, TSD, FCPA and ZLPS 
performed the experiments. TSD, FCPA and ZLPS analyzed the data. GJC and TCP wrote the manuscript and all authors read and approved the final version.

\section{References}

Abe N, Abe H and Ito Y (2012) Synthesis of dumbbell-shaped cyclic RNAs for RNA interference. Curr Protoc Nucleic Acid Chem 48:16.4.1-16.4.11.

Elbashir SM, Harborth J, Lendeckel W, Yalcin A, Weber K and Tuschl T (2001) Duplexes of 21-nucleotide RNAs mediate RNA interference in cultured mammalian cells. Nature 411: 494-498.

Fire A, Xu S, Montgomery MK, Kostas SA, Driver SE and Mello CC (1998) Potent and specific genetic interfering by double-stranded RNA in Caenorhabditis elegans. Nature 391:806-811.

Ganesh S, Shui X, Craig KP, Park J, Wang W, Brown BD and Abrams MT (2018) RNAi-mediated $\beta$-catenin inhibition prootes $\mathrm{T}$ cell infiltration and antitumor activity in combination with immune checkpoint blockade. Mol Ther 26:2567-2579.

Guerrero-Rubio MA, Hernández-García S, García-Carmona F and Gandiá-Herrero F (2019) Extension of life-span using a RNAi model and in vivo antioxidant effect of Opuntia fruit extracts and pure betalains in Caenorhabditis elegans. Food Chem 2748:840-847.

Guo X, Wang Y, Sinakecitch I, Lei H and Smith BH (2018) Comparison of RNAi knockdown effect of tyramine receptor 1 induced by dsRNA and siRNA in brains of the honey bee, Apis mellifera. J Insect Physiol 111:47-52.

Gvozdeva OV, Gladkih DV, Chernikov IV, Meschaninova MI, Venyaminova AG, Zenkova MA, Vlassov VV and Chernolovska EL (2018) Nuclease-resitant 63-bp trimeric siRNAs simultaneously silence three different genes in tumor cells. FEBS Lett 592:122-129.

Holen T, Amarzguioui M, Wiiger MT, Babaie E and Prydz H (2002) Positional effects of short interfering RNAs targeting the human coagulation trigger Tissue Factor. Nucleic Acids Res 30:1756-1766.

Hussain T, Aksoy E, Caliscan ME and Bakhsh A (2019) Transgenic potatoe lines expressing hairpin RNAi construct of molting-associated EcR gene exhibit enhanced resistance against Colorado potaote beetle (Leptinotarsa decemlinea$t a$, Say). Transgenic Res. 28:151-164.

Jang B, Kim B, Kim H, Kwon H, Kim M, Colas M, Seo Y, Jeong $\mathrm{H}$, Jeong EH, Lee $\mathrm{K}$ et al. (2018) Enzymatic synthesis of self-assembled dicer substrate RNA nanostructures for programmable gene silencing. Nano Lett 18:4279-4284.
Kim DG, Longo M, Han Y, Lundberg P, Cantin E and Rossi JJ (2004) Interferon inducing by siRNAs and ssRNAs synthesized by phage polymerase. Nat Biotechnol 22:321-325.

Kim H, Kim D, Jeong J, Jeon H and Lee JB (2018) Size-controllable enzymatic synthesis of short hairpin RNA nanoparticles by controlling the rate of RNA polymerization. Polymers (Basel) 10:589.

Lourenço AP, Mackert A, Cristino AS and Simões ZLP (2008) Validation of reference genes for gene expression studies in the honey bee, Apis mellifera, by quantitative real-time RTPCR. Apidologie 39:372-385.

Matthiesen J, Lender C, Haferkron A, Fehling H, Meyer M, Matthies T, Tannich E, Roeder T, Lotter H and Brucchaus I (2019) Trigger-induced RNAi gene silencing to identify pathogenicity factors of Entamoeba histolytica. FASEB J 33:1658-1668.

Metwali EMR, Soliman HIA, Fuller MP and Almaghrabi OA (2015) Improving fruit quality in tomato (Lycopersicum esculentum Mill) under heat stress by silencing the vis 1 gene using small interfering RNA technology. Plant Cell Tissue Organ Cult 121:153-166.

Pereira TC, Bittencourt VDP, Secolin R, Rocha CS, Maia IG and Lopes-Cendes I (2007) Strand Analysis, a free online program for the computational identification of the best RNA interference (RNAi) targets based on Gibbs free energy. Genet Mol Biol 30:1206-1208.

Seyhan AA, Vlassov AV and Johnston BH (2006) RNA interference form multimeric shRNAs generated by rolling circle transcription. Oligonucleotides 16:353-363.

Shopsowitz KE, Wu C, Liu G, Dreaden EC and Hammond PT (2016) Periodic-shRNA molecules are capable of gene silencing, cytotoxicity and innate immune activation in cancer cells. Nucleic Acid Res 44:545-557.

Suzuki H, Spengler RM, Grigelioniene G, Kobayashi T and Sharp TA (2018) Deconvolution of seed and RNA-binding protein crosstalk in RNAi-based funciontal genomis. Nat Genet 50:657-661.

Wang X, Li C, Gao X, Wang J and Liang X (2015) Preparation of small RNAs using rolling circle transcription and site-specific RNA disconnection. Mol Ther Nucleic Acids 4:e215.

Wang S, Wang Z, Li L, Zou L, Gong Y, Jia T, Zhao S, Yuan H, Shi L, Liu S et al. (2018) P2Y12 shRNA treatment decreases SGC activation to relieve diabetic neurophatic pain in type 2 diabetes mellitus rats. J Cell Physiol 233: 620-9628.

Wu C, Shopsowitz KE and Hammond PT (2016) Engineering periodic shRNA for enhanced silence efficacy. Mol Ther 24:1070-1077.

Associate Editor: Rogério Margis

License information: This is an open-access article distributed under the terms of the Creative Commons Attribution License (type CC-BY), which permits unrestricted use, distribution and reproduction in any medium, provided the original article is properly cited. 